\title{
Assessment of the Small Signal Stability of the European Interconnected Electric Power System Using Neural Networks
}

\author{
S.P. Teeuwsen, I. Erlich, A. Fischer \\ University of Duisburg \\ Germany
}

\begin{abstract}
This paper deals with a new method based on neural networks for eigenvalue predictions of critical stability modes of power systems. Our special interest is focused on inter-area oscillations in the European interconnected power system. The existing methods for eigenvalue computations are time-consuming and require the entire system model that includes an extensive number of states.
\end{abstract}

Keywords: Inter-Area Oscillations, Neural Networks, Small Signal Stability

\section{Introduction}

The European interconnected power system, also known as UCTE/CENTREL, consists of the western European Union for the Coordination of Transmission of Electricity (UCTE) and the central European power system (CENTREL). The CENTREL system includes the central European countries such as Poland, Hungary, and the Balkan States. Due to the recent integration of the CENTREL power system, the European network has grown rapidly. Figure 1 shows a map

\author{
M. A. El-Sharkawi \\ University of Washington \\ Seattle, USA
}

of Europe with 29 different network groups representing UCTE/CENTREL. Table 1 identifies these net groups.

Table 1 Identification of the 29 Net Groups in the European Interconnected Power System

\begin{tabular}{|l|l|l|l|}
\hline ALB & Albania & MAZ & Macedonia \\
\hline B & Belgium & NL & The Netherlands \\
\hline BAG & Germany & OEVG & Austria \\
\hline BEWAG & Germany & PE & Germany \\
\hline BG & Bulgaria & PL & Poland \\
\hline BiH & Bosnia/Herzegovina & Po & Portugal \\
\hline CEZ & Czech Republic & ROM & Romania \\
\hline CH & Switzerland & RWE & Germany \\
\hline ELSAM & Denmark & SEP & Slovakia \\
\hline EnBW & Germany & SL_HR & Slovenia/Croatia \\
\hline FR & France & Sp & Spain \\
\hline GR & Greece & VEAG & Germany \\
\hline HU & Hungary & VEW & Germany \\
\hline IT & Italy & YU & Yugoslavia \\
\hline LVOV & Ukraine & & \\
\hline
\end{tabular}

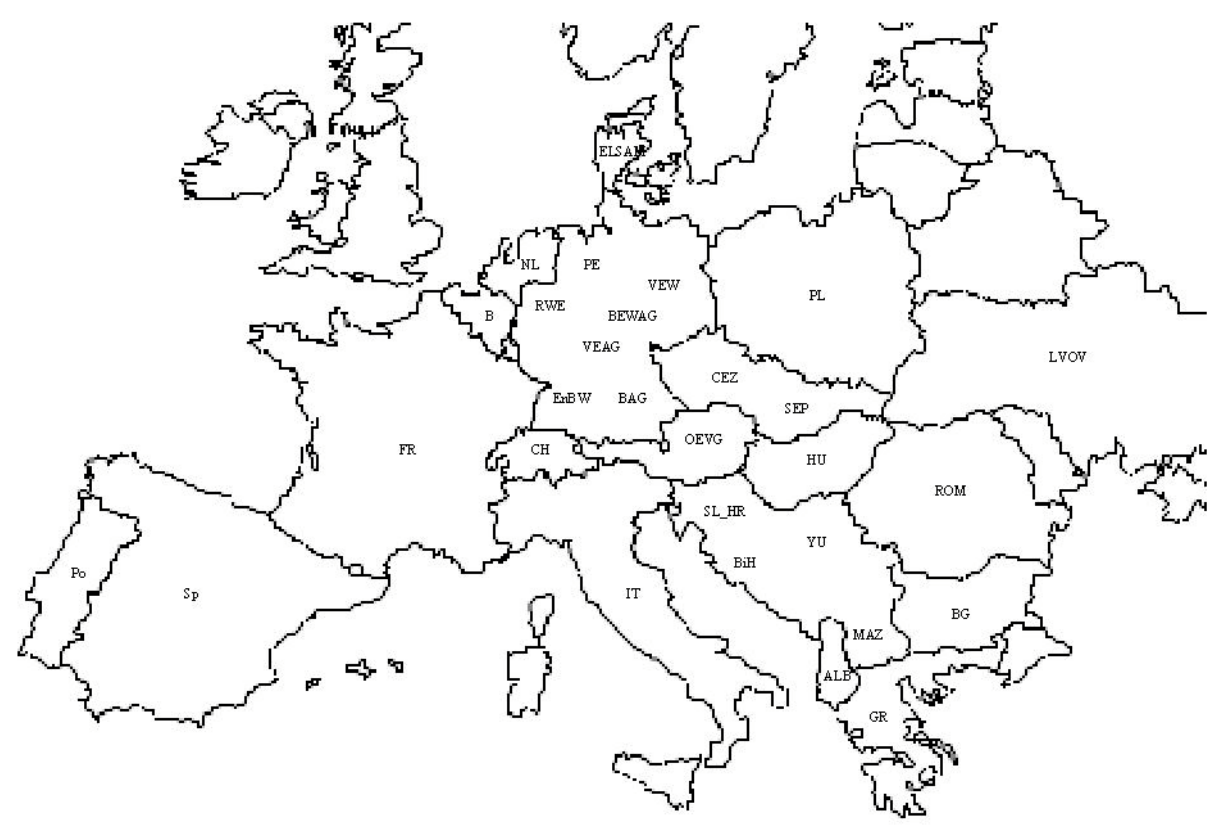

Figure 129 Net Groups in Europe's UCTE/CENTREL Interconnected Power System 
The integration of the two large power systems (UCTE and CENTREL) led to a different stability behavior. Although the European network is strongly mashed, it includes parts with high power concentration, which could swing against each other. Inter-area oscillations are observed when two or more net groups in the power system (i.e. power supply companies) exchange energy. These so-called inter-area oscillations are slow damped oscillations with quite low frequencies. In the European system, small-signal stability is largely a problem of insufficient damping of oscillations $[3,4]$.

With the privatization/liberalization of electric utilities in Europe, the utilities are allowed to sell their generated power outside their traditional borders and compete directly for customers. For economical reasons, the operators are often forced to steer the system closer to the stability limits. Thus, the operators need different computational tools for system stability. These tools must be accurate and fast to allow for on-line stability assessment.

The small-signal stability method (modal analysis) is based on the eigenvalues method [5]. The inter-area modes are associated with the swinging of many machines in one part of the system against machines in other parts. In the European case, three global modes (eigenvalues) are of particular importance because when they lack damping the system starts to oscillate. For example, the status of the load flow that includes Spain, Portugal, Poland or some Balkan States leads very often to an under-damped power system.

\section{Proposed Approach}

The computation of the small signal stability is a time consuming process for large networks, which includes the load flow computation, the linearization at the operating point and the eigenvalue computation. Thus, it is time consuming and not suitable for on-line applications.

An alternative method is to use a neural network (NN) trained with off-line data for different load flow conditions. By using $\mathrm{NN}$, a fast computation of the eigenvalues is possible, providing that the network is properly designed. For on-line applications, the NN predicts the dominant eigenvalues based on the current operating conditions.

Another advantage of the $\mathrm{NN}$ is that it can be properly trained with fewer input features. This is also important considering that due to increasing competition utilities may not share essential information. Only very few features are commonly available such as the transmitted power or the generation of the net groups. Information about single generators or transmission lines is not usually available.

\section{Feature Selection for NN Inputs}

To properly train a neural network, a set of features or measurements from the interconnected power system must be selected. These features like voltages, real power and reactive power of generators and transmission lines must be highly correlated with the dominant eigenvalues.

In this study, the available data for the UCTE/CENTREL system include features for power equipments such as the transmission lines, transformers, generators and loads. The generator model is either $5^{\text {th }}$ or $6^{\text {th }}$ order including AVR and governor models. Hence, there is a large number of features in such extensive power system. The size of this feature set creates the bottleneck problem for $\mathrm{NN}$ training. Hence, feature extraction or selection must be implemented. In this study, the feature selection is performed by engineering judgment, whereby only the available and measurable features are used.

The selected features in this study are:

- Generated real power in each net group

- Generated reactive power in each net group

- Real power transmitted between neighboring net groups

- $\quad$ Reactive power transmitted between neighboring net groups

The generated real and reactive power in each net group is the summation of all generated power within this net group.

Power flow between neighbored net groups is the sum of power of all transmission lines between the two net groups. This leads to $62 \mathrm{NN}$ inputs, which is the number of net group connections.

Generator voltage are not used in this study, because the load flow is based on PV nodes that provide a constant voltage level.

\section{Generation of Patterns for NN Training}

Each computation of a new pattern is for one load flow condition. The basic challenge is to simulate load flow cases that are highly correlated with system stability. To find these cases, a number of off-line computations for each net group are generated. To generate the load flow data, the generators are selected to correspond to one net group.

By simultaneously changing the power in two net groups, the load flow between these two net groups is changed accordingly. The computed eigenvalues under different load flows are shown in Figure 2, and 3.

Figure 2 shows 10 eigenvalues in the complex plain. These eigenvalues are interesting, because they show a low frequency component and are inter-area eigenvalues. The lines in the figure are the borders of $0 \%$ to $20 \%$ damping. Some of the eigenvalues remain in the stable region (eigenvalues 4-10) and are non-dominant, while others (eigenvalues 1,2 and 3) are close to the low damping region and can cause system instability. The 3 dominant 
eigenvalues, selected from Figure 2, are shown separately in Figure 3.

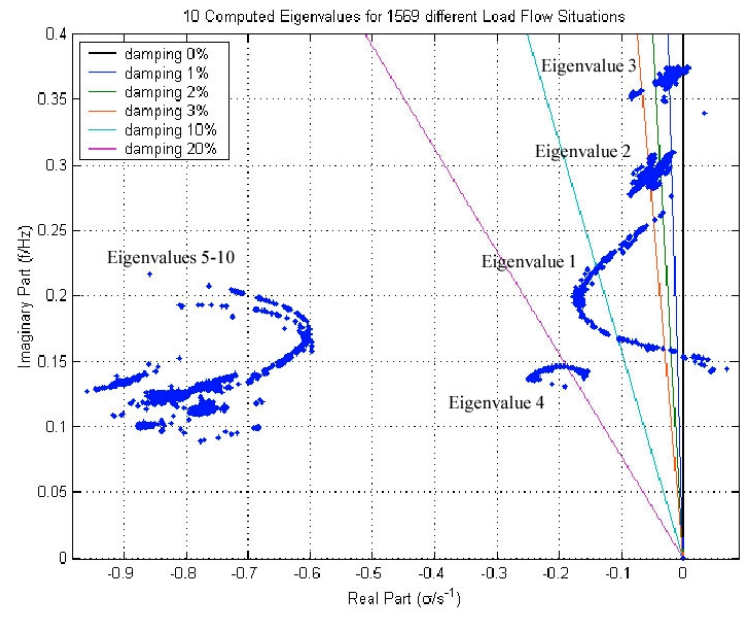

Figure 210 Computed Eigenvalues under 1,569 Different Load Flow Situations

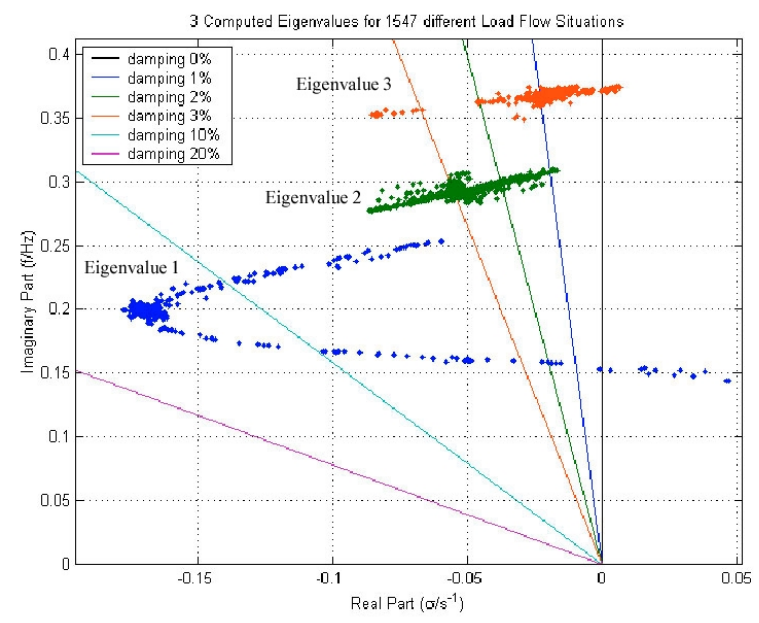

Figure 3 Changes of Dominant Eigenvalues under 1,547 Different Load Flow Situations

Figures 4 to 6 show the mode shape characteristics for the 3 distinguished eigenvalues in the European power system under one load flow situation. The mode shape is computed by using modal analysis [5]. The original state variables are projected onto an equivalent space using the eigenvector multiplication. The eigenvector corresponding to one eigenvalue is represented by the magnitudes and angles of its elements. The magnitude represents the extent of the activities of the equivalent state. The angle represents the phase displacement of the equivalent state variable. The figure illustrates the elements of the eigenvectors by arrows. In Figure 4, it is shown that for one eigenvalue, most of Europe is swinging against the southwest region. The swinging frequency in this case is $0.13 \mathrm{~Hz}$ which corresponds to eigenvalue 1 .

Figure 5 shows, for the same load flow and another eigenvalue, the northeast part of Europe is swinging against the southeast. For this eigenvalue, the frequency of oscillation is $0.36 \mathrm{~Hz}$ which corresponds to eigenvalue 2 . Similarly, Figure 6 shows the oscillations due to eigenvalue 3 . In this case, there are no much oscillations between the net groups. Most of the oscillations are coherent within the southeast region.

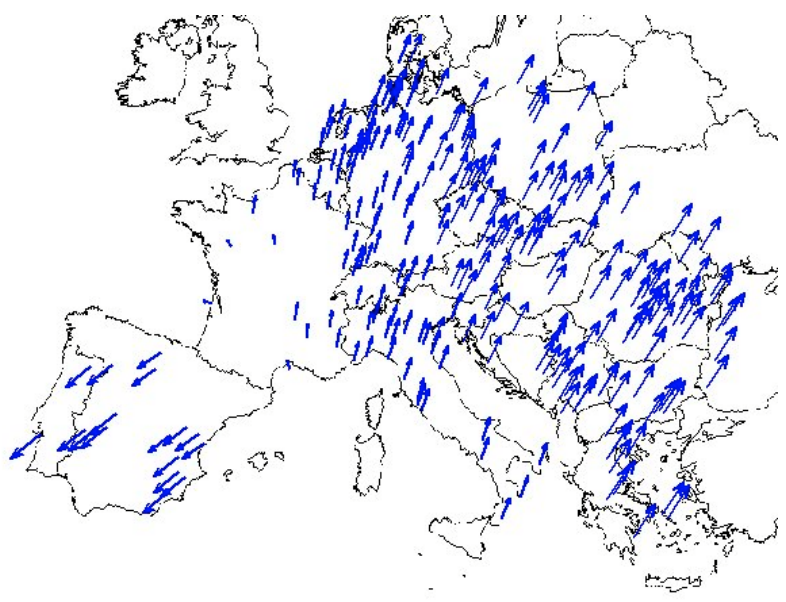

Figure 4 Mode Shape Characteristic for $0.13 \mathrm{~Hz}$

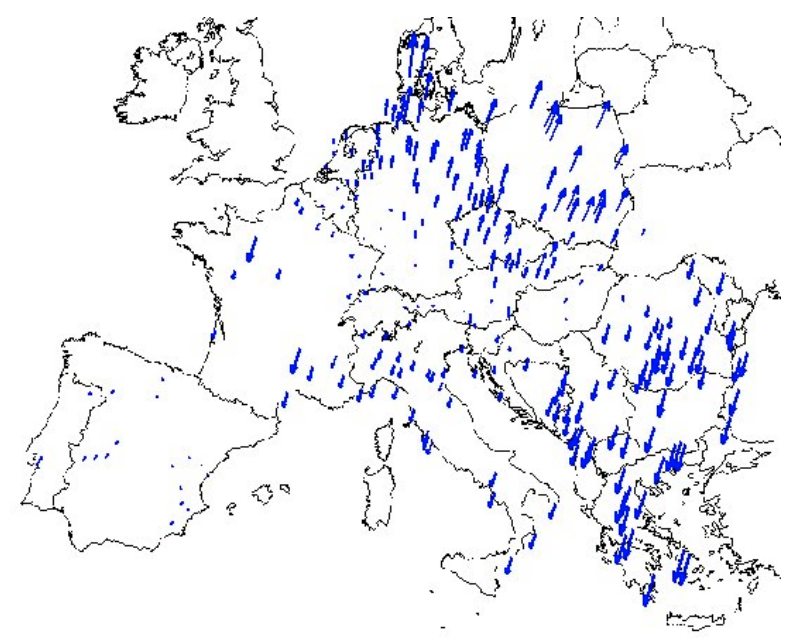

Figure 5 Mode Shape Characteristic for $0.36 \mathrm{~Hz}$

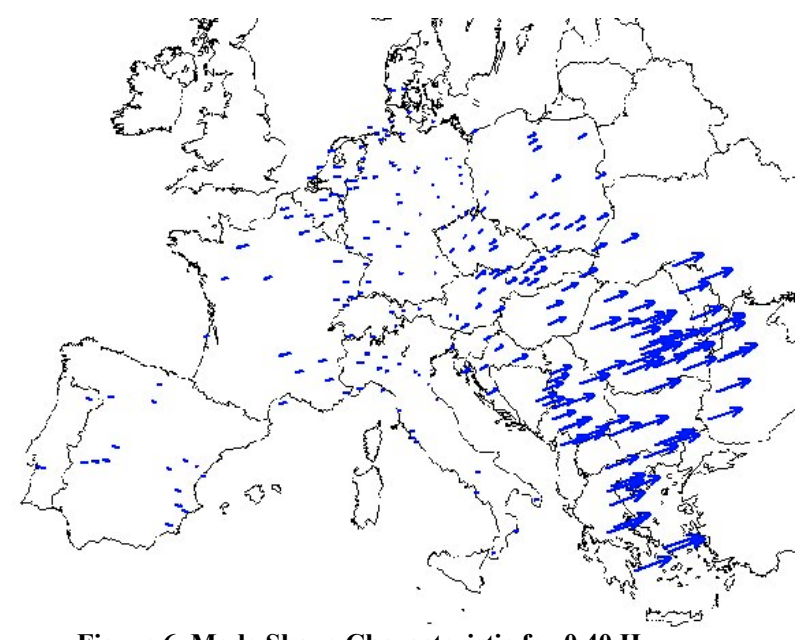

Figure 6 Mode Shape Characteristic for $0.40 \mathrm{~Hz}$ 


\section{Neural Network Structure}

The neural network used in this study is a multilayer feedforward network. The number of inputs depends on the size of the feature set. The number of outputs is 6 for the real and imaginary parts of 3 selected eigenvalues.

The network is trained by the backpropagation algorithm.

During the NN training, the error function decreases but could be trapped in a local minimum. To improve the training, the training error is observed. When the error decreases below a defined rate, the weights are randomly perturbed.

Another tendency of neural networks behavior is memorization. This is an over fitting of training data [1]. Usually, the training error is very small in this case, but the error for the testing patterns is a much larger. To prevent a $\mathrm{NN}$ from memorizing the training data, the training patterns are shuffled after several iterations. The accuracy of the NN during testing is evaluated. The training process is stopped when the testing error starts to substantially increase.

\section{Training Results}

The training data used in this study is shown in Figure 7. The training data consists of 62 transmitted real power. Figure 8 shows the results of the testing stage. 900 patterns are used during training and 100 during testing.

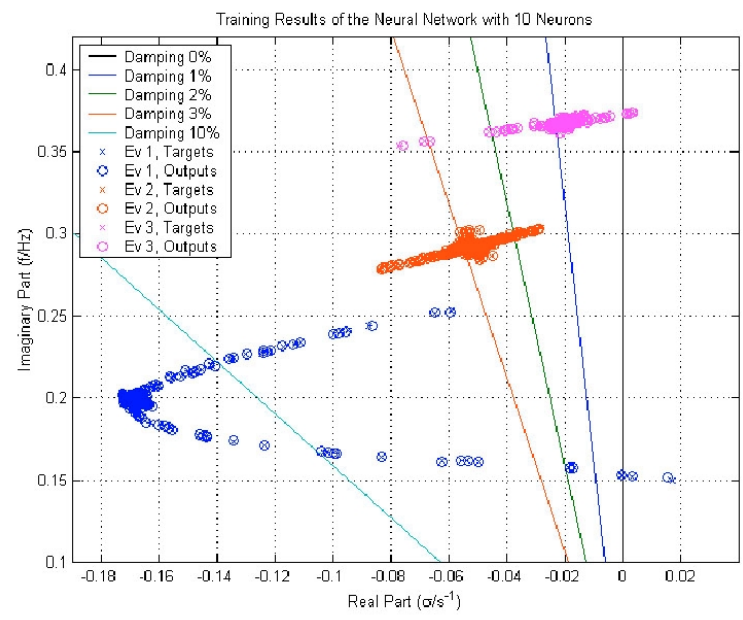

Figure 7 Training data and Results of the Neural Network for 3 Dominant Eigenvalues

The eigenvalues marked with crosses are the ones used as targets. The circles are the $\mathrm{NN}$ outputs.

The targets and the NN outputs are connected by lines.

Figures 9 and 10 show the training and testing results when transmitted reactive power is used. The tests show that the neural network is able to predict the dominant eigenvalues with good accuracy. Moreover, once trained, the NN computes the dominant eigenvalues within milliseconds. In contrast, a computation of eigenvalues including the load flow computation and the linearization takes up to 20 minutes.

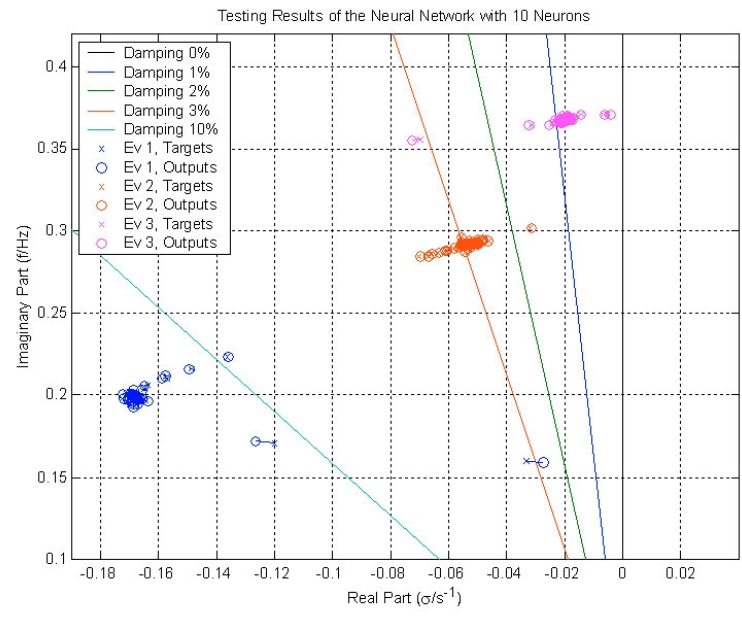

Figure 8 Testing Results of the Neural Network for 3 Dominant Eigenvalues

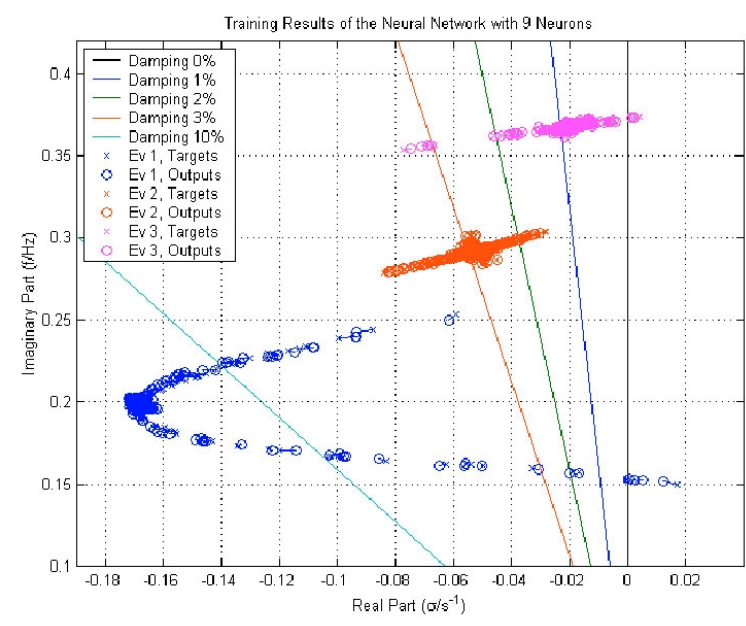

Figure 9 Training Results of the Neural Network for 3 Dominant Eigenvalues

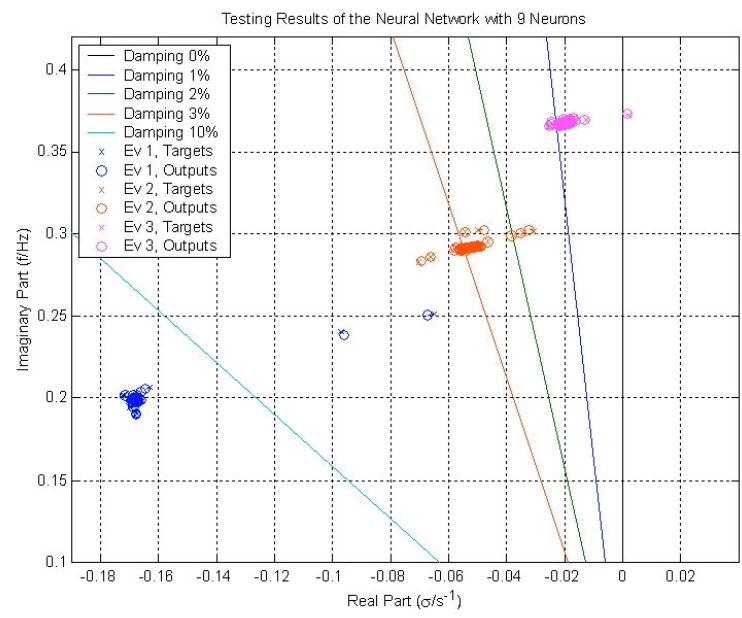

Figure 10 Testing Results of the Neural Network for 3 Dominant Eigenvalues 


\section{Conclusions}

It is shown that only 62 available features for the entire European power system can be used to predict the small signal stability. The NN, when trained by the selected features, can provide on-line calculation of the dominant features of the system with high accuracy.

\section{References}

[1] M.A. El-Sharkawi, "Neural network and its ancillary techniques as applied to power systems", IEE Colloquium on Artificial Intelligence Applications in Power Systems, 1995, Page(s): 3/1 -3/6

[2] Y. Mansour, E. Vaahedi, M.A. El-Sharkawi, "Dynamic security contingency screening and ranking using neural networks", IEEE Transactions on Neural Networks, Volume: 8 Issue: 4, July 1997, pp. 942-950

[3] U. Bachmann, I. Erlich and E. Grebe, "Analysis of interarea oscillations in the European electric power system in synchronous parallel operation with the Central-European networks", IEEE PowerTech, Budapest 1999

[4] H. Breulmann, E. Grebe, M. Lösing, W. Winter, R. Witzmann, P. Dupuis, M.P. Houry, T. Margotin, J. Zerenyi, J. Dudzik, J. Machowski, L. Martín, J.M. Rodríguez, E. Urretavizcaya, "Analysis and Damping of Inter-Area Oscillations in the UCTE/CENTREL Power System", CIGRE 38-113, Session 2000

[5] P. Kundur, Power System Stability and Control, McGraw-Hill, New York, 1994, pp.699-716

\section{Biographies}

Simon P. Teeuwsen (1976) is presently an exchange student at the University of Washington and just finished his diploma thesis about this paper's topic. He started his studies at the University of Duisburg/Germany in 1995. He is a member of VDE and VDI.

Istvan Erlich (1953) received his Dipl.-Ing. degree in electrical engineering from the University of Dresden/Germany in 1976. After his studies, he worked in Hungary in the field of electrical distribution networks. From 1979 to 1991, he joined the Department of Electrical Power Systems of the University of Dresden again, where he received his $\mathrm{PhD}$ degree in 1983. In the period of 1991 to 1998 , he worked with the consulting company EAB in Berlin and the Fraunhofer Institute IITB Dresden respectively. During this time, he also had a teaching assignment at the University of Dresden. Since 1998, he is Professor and head of the Institute of Electrical Power Systems at the University of Duisburg/Germany. His major scientific interest is focused on power system stability and control, modelling and simulation of power system dynamics including intelligent system applications. He is a member of VDE and IEEE.

Armin Fischer (1968) received the Dipl.-Ing. degree in Electrical Engineering from the University of Erlangen-Nuernberg/Germany. Since 1998 he worked as a research associate in the Institute of Electric Power Systems of the University of Duisburg University/Germany. His main research areas are in the modeling and simulation of large-scale power systems.

Mohammed A. El-Sharkawi received the B.Sc. degree in electrical engineering in 1971 from Cairo High Institute of Technology, Egypt, and the M.A.Sc. and Ph.D. degrees in electrical engineering from the University of British Columbia, Vancouver, B.C., Canada, in 1977 and 1980, respectively. In 1980, he joined the University of Washington, Seattle, as a Faculty Member. He served as the Chairman of Graduate Studies and Research and is presently a Professor of Electrical Engineering. He co-edited an IEEE tutorial book on the applications of neural network to power systems. He organized and taught several international tutorials on intelligent systems applications, power quality and power systems, and he organized and chaired numerous panel and special sessions in IEEE and other international conferences. He published over 120 papers and book chapters in these areas and holds seven licensed patents. He is a member of the editorial board and
Associate Editor of several journals, including the IEEE TRANSACTIONS ON NEURAL NETWORKS and Engineering Intelligent Systems. 